\title{
One plus one is better than two. The best choice for embryo transfer: A retrospective cohort study
}

\author{
Pedro Monteleone ${ }^{1}$, Tatiana Bonetti ${ }^{2}$, Sergio Gonçalves ${ }^{1}$, Pedro Peregrino ${ }^{1}$, Alecsandra \\ Gomes $^{1}$, Hamilton de Martin ${ }^{1}$, José Maria Soares ${ }^{3}$, and Edmund Baracat ${ }^{3}$ \\ ${ }^{1}$ Monteleone Human Reproduction Centre \\ ${ }^{2}$ Universidade Federal de São Paulo \\ ${ }^{3}$ Universidade de Sao Paulo Faculdade de Medicina
}

August 28, 2020

\begin{abstract}
Objective: To assess the freeze-only strategy associated with frozen-thawed single-embryo transfers (SETs) efficiency in highresponder patients. Design: Retrospective cohort study Setting: Private reproductive medicine center. Population: A total of 500 IVF cycles with freeze-all embryos followed by elective frozen-thawed embryo transfers (eFET). Method: Elective doubleembryo transfer (eDET) group with 291 cycles, in which two embryos were placed in the first eFET, and elective-SET (eSET) group with 209 cycles, in which the patients underwent eSET in their first eFET. For those who did not become pregnant, a second SET was performed $(n=60)$. Main outcome measures: The ongoing pregnancy rate $(\mathrm{PR})$ was compared after the first FET, and cumulative ongoing PR was evaluated for the SET group considering patients who had a second frozen-thawed SET $($ eSET + SET). Results: No significant differences were observed in the ongoing PR after the first FET (eDET: $37.8 \%$ versus eSET: $34.8 \%, \mathrm{p}=0.497)$. The estimated cumulative ongoing PR for eSET + SET (52.1\%) was significantly higher than eDET $(37.8 \%, \mathrm{p}<0.001)$. The linear multiple regression confirmed that transfer of two embryos in sequential SETs led to a higher chance of implantation than did the transfer of two embryos together (eDET; coefficient: 0.142, p < 0.001). The eDET group had $26.9 \%$ twin pregnancies compared with $1.9 \%$ in the eSET $(\mathrm{p}<0.001)$. Conclusions: Sequential SETs in freeze-only cycles may be the best option to attain high success rates in high-responder patients, leading to an increased chance of implantation and avoidance of multiple gestations.
\end{abstract}

\section{Introduction}

Since the first successful conception via in vitro fertilization (IVF) 40 years ago, advances in protocols have resulted in increasing success rates. Among them, improvements in culture conditions that allow a higher number of fertilized oocytes to reach the blastocyst stage and the development of vitrification techniques with excellent frozen-thawed blastocyst survival rates with no impact on the implantation potential are the most important. Nowadays, in the era of personalized medicine, the practice of fixed protocols is becoming outdated and defining individualized parameters for each situation is now considered more appropriate for obtaining higher success rates (i.e., ongoing pregnancy rates [PRs]) and fewer adverse effects (i.e., multiple pregnancies) of IVF.

These advances have allowed the evolution of two practices in assisted reproductive technology (ART): freezeonly strategy and single-embryo transfer (SET). The freeze-only strategy, in which all available good-quality embryos are frozen and transfers are delayed for a more physiologic cycle (natural or hormone replacement cycle), has been increasingly used. Previous studies have suggested that frozen-thawed embryo transfers 
(FETs) in the absence of ovarian stimulation allow better synchrony between blastocyst and endometrium maturation (1), which is an essential step in the interaction between an implantation-competent blastocyst and a receptive endometrium $(2,3)$. Hence, transferring embryos in a more physiological uterine environment could hypothetically improve the overall outcomes (4). The freeze-only strategy is being used for patients at a risk for ovarian hyperstimulation syndrome (OHSS) (5) and those undergoing preimplantation genetic testing for aneuploidy (6). However, there is no evidence supporting the widespread use of the freeze-only strategy for all patients undergoing IVF and a number of additional questions need to be answered (7-9).

The SET is another practice who have been increasing applied after the improvement in embryo vitrification techniques. SET is the best choice to reduce multiple pregnancies and the associated risks, as preterm labour and birth, gestational hypertension, gestational diabetes, premature birth, premature rupture of membranes, etc (10-12). However, SET is not always practiced, although there is recommendations for a reduction in the number of embryos transferred (ET) (13). Double embryo transfer (DET) is the most common practice worldwide and multiple pregnancies remain the most important iatrogenic complication of assisted reproductive technology (ART) (14). Efforts has been towards to stimulate SET. Studies demonstrate the transfer of two embryos in sequential SET cycles results in similar cumulative live birth rates compared to DET, with reduced multiple pregnancy $(15,16)$. Studies from our group also confirmed that SET is a valuable practice for good prognosis patients (17-19), as those younger than 38 years of age, good ovarian response (at least 4 oocytes collected) and without severe male factor $(20,21)$.

Based on the theory that patients at risk of OHSS should undergo freeze-only approach and at the same time the increased number of oocytes characterize those patients as good prognosis, which is one of the indications for SET, we hypothesized that high responder patients undergoing freeze-only strategy to avoid the OHSS, the use of consecutive elective single embryo transfers can result in the most efficient approach in terms of higher pregnancy success rates and less complications. To answer our question, we assessed retrospectively the clinical outcomes of IVF cycles of high responder patients who underwent freeze-only strategy and SET in the last 9 years in our centre. As control group, we included patients with the same characteristics who underwent freeze-only strategy and DET.

\section{Methods}

This was a retrospective cohort study evaluating freeze-only cycles performed in a private IVF centre in Sao Paulo, Brazil. All procedures in this study are part of the routine care in the assisted reproductive centre and written informed consent was obtained from all patients before treatment, consenting to the treatment procedures and to the use of their data in scientific publications with no patient identification. This study is based on databank of anonymized data and according to Brazilian legislation it was exempt from approval by the Institutional Review Board and specific Informed Consent is not applicable.

The database included all IVF cycles performed between 2011 and 2019 at Monteleone Assisted Reproduction Center, São Paulo - Brazil, that were potentially eligible for this study. The inclusion criteria were cycles of patients in which all embryos were cryopreserved (freeze-only cycles) and no fresh embryo transfer were placed. From 5156 cycles performed in the period of study, we selected 2725 freeze-only cycles. From those, we excluded cycles using donated oocytes, testicle sperm, embryo biopsy, cycles with less than three embryos cryopreserved and more than 2 embryos transferred in the frozen-thawed ET. Missing data were not a reason for case exclusion, and all cycle analysed had all essential data (associated to inclusion or exclusion criteria) and the most of other additional information. Thus, missing data did not compromised the analysis. The final number of 500 freeze-only cycles with elective Frozen-Thawed Embryo Transfer (eFET) of one or two embryos was analysed. The cycles were split into two study groups where the elective Double Embryo Transfer group (eDET group) was composed by 291 cycles in which two embryos were placed in the first eFET and had least one surplus embryo cryopreserved and the elective Single Embryo Transfer group (eSET group) with 209 cycles in which patients underwent a eSET in their first eFET and had least one surplus embryo 
cryopreserved. For those who underwent eSET and did not become pregnant, a second frozen-thawed SET was performed for 60 patients (Figure 1).

\section{IVF protocol}

Patients underwent ovarian stimulation and oocyte pickup according to routine medical criteria. Briefly, pituitary blockage was obtained with a GnRH antagonist (Cetrotide? , Merck). Ovarian stimulation was accomplished using recombinant FSH ( $\mathrm{rFSH}$, Gonal-F?, Merck) at $150 \mathrm{IU} /$ day as the starting dose for women up to 35 years of age and $225 \mathrm{IU} /$ day for women older than 35 years and the dose was adjusted according to the ovarian response. Follicular maturation was triggered when at least two follicles reached a diameter of $18 \mathrm{~mm}$ by using a GnRH agonist (Gonapeptyl, Ferring). Oocyte retrieval was performed after 35 to $36 \mathrm{~h}$ by transvaginal ultrasound-guided aspiration. All oocytes were fertilized by ICSI (22) according to routine procedures and embryos were cultured using standard methods in a triple gas incubator $\left(90 \% \mathrm{~N}_{2}\right.$, $5 \% \mathrm{O}_{2}$ and $\left.6 \% \mathrm{CO}_{2}\right)$ at $37 \mathrm{deg} \mathrm{C}$ until vitrification.

All good quality embryos were vitrified on D3 or D5 using the Vitrification Freeze kit (Irvine Scientific) with a Cryotip device (Irvine Scientific), following the manufacturer's instructions. For warming, a Vitrification Thaw kit (Irvine Scientific) was used. Embryos were evaluated by morphological criteria on day 3 (D3) and/or day 5 (D5). The embryos on D3 were considered good quality when they presented 8 to 10 symmetric blastomeres, no multinucleation and a maximum fragmentation level of $20 \%$ (23). Blastocysts on D5 were considered good quality when they were expanded, inner cell mass grade 3 or 4 and the trophectoderm was classified as A or B (24).

For frozen-thawed embryo transfers, endometrial preparation was conducted with $100 \mu \mathrm{g}$ of oestradiol valerate (Estradot, Novartis) for 14 days plus $800 \mathrm{mg}$ of vaginal micronized progesterone (Utrogestan, Farmoquimica) beginning 5 days before the transfer. Embryos were warmed and evaluated for survival and morphology and a higher quality blastocyst was preferentially transferred when available. Clinical pregnancy was defined by the presence of a gestational sac with heartbeat at 2 weeks after biochemical confirmation of pregnancy with serum beta-hCG measurement.

\section{Data collection and statistical analysis}

Data were obtained from the clinical report forms and tabulated for this study. The primary endpoint was the ongoing pregnancy defined by the presence of a gestational sac with heartbeat and the ongoing pregnancy rate $(\mathrm{PR})$ was calculated as the number of patients presenting an ongoing PR divided by the number of patients with embryos transferred. Additionally, for the calculation of the cumulative ongoing PR considering the $2^{\text {nd }}$ SET for patients who did not become pregnant in the $1^{\text {st }}$ SET (eSET-SET group), we used a formula previously described by Luke and colleagues (2015). The cumulative ongoing PR was equal to [ongoing PR for the $1^{\text {st }}$ SET + the ongoing PR for the $2^{\text {nd }}$ SET $*\left(1\right.$ - the ongoing PR for the $1^{\text {st }}$ SET)]. This calculation assumes no contraindication during cycle 1 for continuing into cycle 2 . The implantation rate (IR) was calculated as the number of gestational sacs divided by the number of embryos transferred and miscarriage rate was defined as number of miscarriage divided by the number of patients with gestational sac.

Data analysis was performed using SPSS V.21 (IBM SPSS Software, USA). Normality distribution tests were performed and patient demographic data were evaluated using descriptive statistics, including the means and frequencies. As data were normally distributed, parametric tests to compare means (Student's t test) were used to continuous variables. Pearson's chi-squared test was used to compare frequencies as appropriated. Regression analysis was used to evaluate the associations between variables, and multivariate models included possible confounders. We considered p-values [?]0.05 to be statistically significant. 


\section{Results}

The demographic characteristics of the patients/cycles included in the study are presented in Table I. The variables were compared using Student's t-test, and despite the infertility time, the number of total and metaphase II oocytes collected had statistically significant differences, the groups were clinically comparable.

\section{Clinical outcomes}

For the first FET, we compared the clinical outcomes of the eDET and eSET groups (Table II). The eDET group had a significantly higher multiple PR and a significantly lower implantation rate, as expected, and the ongoing PRs were similar between the two groups. Conversely, when a second SET was performed in patients who did not become pregnant in the first frozen-thawed eSET (eSET + SET, $\mathrm{n}=60$ ), 16 ongoing pregnancies $(26.7 \%)$ were achieved in this group. Thus, the estimated cumulative ongoing PRs of the eSET + SET subgroup were calculated according to the previously described formula, and became significantly higher from those of the eDET group (Figure 2).

The multiple linear regression model evaluated the association of transferring two embryos in two sequential transfers (eSET + SET), compared with the eDET protocol, with the chance of embryo implantation. The model was adjusted for confounders such as the women's age and the number of cryopreserved embryos. We obtained a statistically significant model, in which the transfer of two embryos in two sequential transfers $(\mathrm{eSET}+\mathrm{SET})$ led to a significantly higher chance of implantation than did the transfer of two embryos together (eDET) in freeze-only cycles (coefficient: 0.142, $\mathrm{p}<0.001)$, adjusted for women's age and number of cryopreserved embryos available (Table III).

\section{Discussion}

This study aimed to evaluate the success rates of two consecutive SETs in patients with a good prognosis compared with the transfer of two embryos in one frozen-thawed cycle, and demonstrated that the first approach is superior in terms of ongoing PRs. The strategy of transferring embryos one by one results in higher ongoing PRs with the advantage of avoiding multiple gestation. Although the use of a freeze-only strategy in patients at a risk for OHSS has been described $(5,25-27)$, studies on the freeze-only strategy have compared the method with fresh transfers, and no consensus has been reached thus far $(8,9,28,29)$.

The association of the freeze-only strategy with subsequent consecutive frozen-thawed SETs has not been extensively studied. A study evaluating the association between the freeze-only strategy and SET in women with hypogonadotropic hypogonadism showed that SET is an effective strategy for decreasing the incidence of multiple conceptions while maintaining satisfactory live birth rates (50.5\%) (30). He and colleagues compared single and double frozen-thawed blastocyst transfers after a freeze-only strategy and found similar cumulative PRs (31). Our study found fewer multiple pregnancies with eSET, as expected; however, we also observed that two consecutive frozen-thawed SETs resulted in higher ongoing PRs than the transfer of two blastocysts in one frozen-thawed cycle. The regression model also confirmed an association of two consecutive SETs with a higher chance of implantation, which may indicate that transferring blastocysts one by one in a freeze-only strategy is better than transferring two blastocysts together.

A number of variables are associated with the embryo implantation potential, and they have been the focus of several studies. Not only the embryo quality evaluated using conventional morphology, time-lapse morphokinetics, or preimplantation genetic test (32) but also the endometrium status (33) and embryo-endometrial synchrony are crucial factors for a successful implantation (34). Studies from Simon and collaborators have investigated the progesterone action, endometrium gene, receptors, and protein expression based on data from the endometrial receptivity array (35-39). More recently, the endometrium microbiome and its association with embryo implantation have been studied by the same research group (40-42). Although the association of endometrium gene expression or microbiome with the implantation rates is controversial in the 
literature (43), it is clear that there is a variability in the endometrial condition between cycles according to the patient's clinical condition or changes in the treatment approaches. These variations justify the greater chance of implantation when performing consecutive SETs compared with one DET. However, the large diversity of variables involved in embryo implantation, with respect to both the embryo and endometrium, makes the complete elucidation of that process impossible.

The vitrification technique is able to maintain the embryo implantation potential $(44,45)$, which, in turn, allows the performance of a freeze-only strategy and endometrium preparation for embryo transfer in natural or hormone replacement cycles. This was confirmed by an extensive study evaluating $>20,000$ freezeonly cycles and FETs, in which all embryos transferred from that stimulation cycle were considered. The study demonstrated that the mean cumulative live birth rate was $50.0 \%$ and dependent on the number of oocytes collected and the patient's age, suggesting the applicability of the freeze-only strategy for the general population (46). However, that study did not consider the number of embryos transferred, which allowed us to evaluate the association of the freeze-only strategy with SET. Also confirming our hypothesis of better clinical outcomes after a freeze-only strategy plus SET, the American Society for Reproductive Medicine and the Society for Assisted Reproductive Technology (ASRM/SART) recommended SET for patients with a good prognosis aged $<38$ years, except in cases in which the patient had previously experienced several cycle failures and for whom DET was suggested (47).

Another study evaluated retrospective data about clinical PRs before and after the ASRM/SART guidelines, as their clinic policy is to transfer no more than the recommended number of embryos. The study assessed patients $<38$ years old using their own eggs and without preimplantation genetic testing, before (mean of 1.3 embryos transferred per patient) and after (all embryos transfers were single) guideline revision. The outcomes showed that SET was very efficient in this population, as the overall live birth rates were maintained at around $50 \%$ after the reduction in the number of embryos transferred, and the twin PR decreased from $14.2 \%$ to $2.5 \%$ (48). This study also supported our hypothesis of the considerable advantages of transferring single embryos, highlighting the absence of reduction in PRs in SET and avoiding multiple pregnancies after a reduction in the number of embryos transferred.

Owing to the retrospective nature of our study, we adjusted our statistical analysis for confounders. However, we cannot exclude the possibility of residual interfering factors such as endometrium status or embryo ploidy, as these conditions were not evaluated in the cycles included in our study. A limitation of our study lies in the fact that not all patients who had failed eSET in the first transfer proceeded to a second SET cycle. Nevertheless, the calculation used in our study estimated the cumulative ongoing PRs as if all women who had a failed first SET had a second SET, confirming a clear advantage of transferring consecutive embryos one by one over DET. Another point to consider is that the decision about the number of embryos to be transferred and the embryo quality were not controlled. As a clinical routine, the number of embryos transferred was determined in a shared decision-making process between patients and doctors, after explaining the advantages and disadvantages of each situation. Thus, the choice of the number of embryos to be transferred could have been influenced by the quality of the blastocysts available and the couple's preferences. Although our practice prioritizes transferring the best-quality frozen-thawed blastocysts available, data on the quality of embryos transferred were not available for the analysis. All cycles included were elective (with at least one surplus frozen blastocyst) and the demographic characteristics of our study groups were similar, which made the comparisons possible.

\section{Conclusions}

Our study is important in that it not only shows that eSET in freeze-only cycles maintains similar PRs to those of DET after the first transfer but also that a second consecutive SET brings the best cost-benefit ratio, as it increases the success rates compared with DET and decreases the possibility multiple pregnancies. Considering the existence of variables that are not subject to control in a clinical routine, and those inherent to the procedure that can be corrected in a subsequent cycle, it is reasonable to perceive that the transfer of 
embryos in separate events (consecutive SETs) will allow possible corrections and produce a higher success rate than the transfer of two embryos in a single event. Finally, the freeze-only strategy with consecutive SETs is a feasible and efficient approach leading to an increased chance of implantation and avoidance of multiple gestations.

\section{Acknowledgements}

The authors gratefully acknowledge the contributions of the team from Centro de Reprodução Humana Monteleone, São Paulo, Brazil, for their excellent support with patients, procedures, and data collection.

\section{Disclosure of interests}

There is no conflict of interest associated to this publication.

\section{Contribution to Authorship}

Pedro AA Monteleone: substantial contributions to conception and design; acquisition of data, analysis, and interpretation of data; drafting the article; and final approval of the version to be published

Tatiana CS Bonetti: substantial contributions to analysis and interpretation of data, drafting the article, and revising the article critically for important intellectual content

Sergio P Gonçalves: data acquisition and revising the article critically for important intellectual content Pedro FM Peregrino: data acquisition and revising the article critically for important intellectual content Alecsandra P Gomes: data acquisition and revising the article critically for important intellectual content Hamilton de Martin: data acquisition and revising the article critically for important intellectual content José Maria Soares: revising the article critically for important intellectual content Edmund C Baracat: revising the article critically for important intellectual content

\section{Ethics Approval}

All of the procedures in this study are part of routine care in the assisted reproductive center. All patients who underwent assisted reproductive treatment signed a written informed consent agreeing with the procedures related to the treatment, as stablished by the Brazilian Federal Medicine Council (Conselho Federal de Medicina ). In addition, patients consented to their data to be used for scientific publication purposes, provided anonymity was respected. Therefore, the study was exempt from approval by the Institutional Review Board.

\section{Funding}

This research did not receive any specific grant from funding agencies in the public, commercial, or not-forprofit sectors. 


\section{References}

1. Shapiro BS, Daneshmand ST, Garner FC, Aguirre M, Hudson C. Clinical rationale for cryopreservation of entire embryo cohorts in lieu of fresh transfer. Fertility and sterility. 2014;102(1):3-9.

2. Cha J, Sun X, Dey SK. Mechanisms of implantation: strategies for successful pregnancy. Nat Med. 2012;18(12):1754-67.

3. Zhang S, Lin H, Kong S, Wang S, Wang H, Wang H, et al. Physiological and molecular determinants of embryo implantation. Mol Aspects Med. 2013;34(5):939-80.

4. Cedars MI. Fresh versus frozen: initial transfer or cumulative cycle results: how do we interpret results and design studies? Fertility and sterility. 2016;106(2):251-6.

5. Davenport MJ, Vollenhoven B, Talmor AJ. Gonadotropin-Releasing Hormone-Agonist Triggering and a Freeze-All Approach: The Final Step in Eliminating Ovarian Hyperstimulation Syndrome? Obstet Gynecol Surv. 2017;72(5):296-308.

6. Cimadomo D, Capalbo A, Levi-Setti PE, Soscia D, Orlando G, Albani E, et al. Associations of blastocyst features, trophectoderm biopsy and other laboratory practice with post-warming behavior and implantation. Human reproduction. 2018;33(11):1992-2001.

7. Roque M, Nuto Nobrega B, Valle M, Sampaio M, Geber S, Haahr T, et al. Freeze-all strategy in IVF/ICSI cycles: an update on clinical utility. Panminerva Med. 2019;61(1):52-7.

8. Roque M, Haahr T, Esteves SC, Humaidan P. The 'Big Freeze': freeze-all should not be used for everyone. Human reproduction. 2018.

9. Coutifaris C. Freeze-only in vitro fertilization cycles for all? Fertility and sterility. 2017;108(2):233-4.

10. Kallen B, Finnstrom O, Nygren KG, Olausson PO. Temporal trends in multiple births after in vitro fertilisation in Sweden, 1982-2001: a register study. BMJ. 2005;331(7513):382-3.

11. Kallen B, Finnstrom O, Lindam A, Nilsson E, Nygren KG, Otterblad Olausson P. Trends in delivery and neonatal outcome after in vitro fertilization in Sweden: data for 25 years. Human reproduction. 2010;25(4):1026-34.

12. Kallen B, Finnstrom O, Nygren KG, Olausson PO. In vitro fertilization (IVF) in Sweden: infant outcome after different IVF fertilization methods. Fertility and sterility. 2005;84(3):611-7.

13. Practice Committee of Society for Assisted Reproductive T, Practice Committee of American Society for Reproductive M. Elective single-embryo transfer. Fertility and sterility. 2012;97(4):835-42.

14. Sunderam S, Kissin DM, Crawford SB, Folger SG, Jamieson DJ, Warner L, et al. Assisted Reproductive Technology Surveillance - United States, 2014. MMWR Surveill Summ. 2017;66(6):1-24.

15. Luke B, Brown MB, Wantman E, Stern JE, Baker VL, Widra E, et al. Application of a validated prediction model for in vitro fertilization: comparison of live birth rates and multiple birth rates with 1 embryo transferred over 2 cycles vs 2 embryos in 1 cycle. Am J Obstet Gynecol. 2015;212(5):676 e1-7.

16. McLernon DJ, Harrild K, Bergh C, Davies MJ, de Neubourg D, Dumoulin JC, et al. Clinical effectiveness of elective single versus double embryo transfer: meta-analysis of individual patient data from randomised trials. BMJ. 2010;341:c6945.

17. Monteleone PAA, Peregrino PFM, Baracat EC, Serafini PC. Transfer of 2 Embryos Using a DoubleEmbryo Transfer Protocol Versus 2 Sequential Single-Embryo Transfers: The Impact on Multiple Pregnancy. Reprod Sci. 2018;25(10):1501-8. 
18. Monteleone PA, Mirisola RJ, Goncalves SP, Baracat EC, Serafini PC. Outcomes of elective cryopreserved single or double embryo transfers following failure to conceive after fresh single embryo transfer. Reproductive biomedicine online. 2016;33(2):161-7.

19. Monteleone PAA, Petersen PGMF, Peregrino PFM, Miorin J, Gomes AP, Fujii MG, et al. Should single embryo transfer to be used for patients with any kind of infertility factor? Preliminary outcomes. 22st Annual Congress of the SBRA; Brasilia, DF: JBRA Assisted Reproduction; 2018. p. 263.

20. Sunkara SK, Chinta P, Kamath MS. Perinatal Outcomes Following Assisted Reproductive Technology. J Hum Reprod Sci. 2019;12(3):177-81.

21. Ata B, Kaplan B, Danzer H, Glassner M, Opsahl M, Tan SL, et al. Array CGH analysis shows that aneuploidy is not related to the number of embryos generated. Reproductive biomedicine online. 2012;24(6):61420.

22. Palermo G, Joris H, Devroey P, Van Steirteghem AC. Pregnancies after intracytoplasmic injection of single spermatozoon into an oocyte. Lancet. 1992;340(8810):17-8.

23. Veeck LL. An atlas of human gametes and conceptuses : an illustrated reference for assisted reproductive technology. New York: Parthenon Pub. Group; 1999. 215 p. p.

24. Gardner DK, Lane M, Stevens J, Schlenker T, Schoolcraft WB. Blastocyst score affects implantation and pregnancy outcome: towards a single blastocyst transfer. Fertility and sterility. 2000;73(6):1155-8.

25. Shin JJ, Jeong Y, Nho E, Jee BC. Clinical outcomes of frozen embryo transfer cycles after freeze-all policy to prevent ovarian hyperstimulation syndrome. Obstet Gynecol Sci. 2018;61(4):497-504.

26. Wu MY, Chung CH, Pan SP, Jou GC, Chen MJ, Chang CH, et al. Advantages of cumulative pregnancy outcomes in freeze-all strategy in high responders - A case-control matching analysis of a large cohort. J Formos Med Assoc. 2018;117(8):676-84.

27. Acharya KS, Acharya CR, Bishop K, Harris B, Raburn D, Muasher SJ. Freezing of all embryos in in vitro fertilization is beneficial in high responders, but not intermediate and low responders: an analysis of 82,935 cycles from the Society for Assisted Reproductive Technology registry. Fertility and sterility. 2018;110(5):8807 .

28. Zaca C, Bazzocchi A, Pennetta F, Bonu MA, Coticchio G, Borini A. Cumulative live birth rate in freezeall cycles is comparable to that of a conventional embryo transfer policy at the cleavage stage but superior at the blastocyst stage. Fertility and sterility. 2018;110(4):703-9.

29. Le KD, Vuong LN, Ho TM, Dang VQ, Pham TD, Pham CT, et al. A cost-effectiveness analysis of freeze-only or fresh embryo transfer in IVF of non-PCOS women. Human reproduction. 2018;33(10):1907-14.

30. Kuroda K, Ezoe K, Kato K, Yabuuchi A, Segawa T, Kobayashi T, et al. Infertility treatment strategy involving combined freeze-all embryos and single vitrified-warmed embryo transfer during hormonal replacement cycle for in vitro fertilization of women with hypogonadotropic hypogonadism. J Obstet Gynaecol Res. 2018;44(5):922-8.

31. He QH, Wang L, Liang LL, Zhang HL, Zhang CL, Li HS, et al. Clinical outcomes of frozen-thawed single blastocyst transfer in patients requiring whole embryo freezing. Syst Biol Reprod Med. 2016;62(2):133-8.

32. Sigalos G, Triantafyllidou O, Vlahos NF. Novel embryo selection techniques to increase embryo implantation in IVF attempts. Arch Gynecol Obstet. 2016;294(6):1117-24.

33. Kliman HJ, Frankfurter D. Clinical approach to recurrent implantation failure: evidence-based evaluation of the endometrium. Fertility and sterility. 2019;111(4):618-28.

34. Teh WT, McBain J, Rogers P. What is the contribution of embryo-endometrial asynchrony to implantation failure? Journal of assisted reproduction and genetics. 2016;33(11):1419-30. 
35. Diaz-Gimeno P, Horcajadas JA, Martinez-Conejero JA, Esteban FJ, Alama P, Pellicer A, et al. A genomic diagnostic tool for human endometrial receptivity based on the transcriptomic signature. Fertility and sterility. 2011;95(1):50-60, e1-15.

36. Garrido-Gomez T, Quinonero A, Antunez O, Diaz-Gimeno P, Bellver J, Simon C, et al. Deciphering the proteomic signature of human endometrial receptivity. Human reproduction. 2014;29(9):1957-67.

37. Garrido-Gomez T, Ruiz-Alonso M, Blesa D, Diaz-Gimeno P, Vilella F, Simon C. Profiling the gene signature of endometrial receptivity: clinical results. Fertility and sterility. 2013;99(4):1078-85.

38. Gomez E, Ruiz-Alonso M, Miravet J, Simon C. Human Endometrial Transcriptomics: Implications for Embryonic Implantation. Cold Spring Harb Perspect Med. 2015;5(7):a022996.

39. Ruiz-Alonso M, Blesa D, Diaz-Gimeno P, Gomez E, Fernandez-Sanchez M, Carranza F, et al. The endometrial receptivity array for diagnosis and personalized embryo transfer as a treatment for patients with repeated implantation failure. Fertility and sterility. 2013;100(3):818-24.

40. Simon C. Introduction: Do microbes in the female reproductive function matter? Fertility and sterility. 2018;110(3):325-6.

41. Moreno I, Simon C. Deciphering the effect of reproductive tract microbiota on human reproduction. Reprod Med Biol. 2019;18(1):40-50.

42. Moreno I, Simon C. Relevance of assessing the uterine microbiota in infertility. Fertility and sterility. 2018;110(3):337-43.

43. Bassil R, Casper R, Samara N, Hsieh TB, Barzilay E, Orvieto R, et al. Does the endometrial receptivity array really provide personalized embryo transfer? Journal of assisted reproduction and genetics. 2018;35(7):1301-5.

44. Rienzi L, Gracia C, Maggiulli R, LaBarbera AR, Kaser DJ, Ubaldi FM, et al. Oocyte, embryo and blastocyst cryopreservation in ART: systematic review and meta-analysis comparing slow-freezing versus vitrification to produce evidence for the development of global guidance. Hum Reprod Update. 2017;23(2):139-55.

45. Zeng M, Su S, Li L. Comparison of pregnancy outcomes after vitrification at the cleavage and blastocyst stage: a meta-analysis. Journal of assisted reproduction and genetics. 2018;35(1):127-34.

46. Zhu Q, Chen Q, Wang L, Lu X, Lyu Q, Wang Y, et al. Live birth rates in the first complete IVF cycle among 20687 women using a freeze-all strategy. Human reproduction. 2018;33(5):924-9.

47. ASRM/SART. Practice Committee of the American Society for Reproductive Medicine and Practice Committee of the Society for Assisted Reproductive Technology. Guidance on the limits to the number of embryos to transfer: a committee opinion. Fertility and sterility. 2017;107(4):901-3.

48. Eubanks AA, Anthony DeAngelis, Mae W. Healy, Rhiana D. Saunders, Saioa Torrealday, Alan H. Decherney, et al. The Continued Push Towards Eliminating Twin Pregnancy: The Clinical Impact of the 2017 ASRM Embryo Transfer Guidelines. Fertility and sterility. 2019;112(3):Supplement, Page e146

\section{Figure legends}

Figure 1: Flow diagram of the study design.

IVF, in vitro fertilization; ICSI, intracytoplasmic sperm injection; eDET, elective double-embryo transfer; eSET, elective single-embryo transfer

Figure 2: Cumulative clinical pregnancy rate after the transfer of two embryos in one transfer (eDET group) and in two single embryo transfers (eSET + SET group).

PR, pregnancy rate; SET, single-embryo transfer; eDET, elective double-embryo transfer

\section{Tables}


Table I: Demographic characteristics of patients and cycles included in the study

\begin{tabular}{llll}
\hline & eDET & eSET & $\mathrm{p}^{\mathrm{a}}$ \\
\hline Age (years) & $34.9 \pm 4.2$ & $35.4 \pm 3.9$ & 0.155 \\
Body mass index $\left(\mathrm{kg} / \mathrm{m}^{2}\right)$ & $23.1 \pm 3.6$ & $22.5 \pm 2.9$ & 0.092 \\
Infertility time (years) & $2.9 \pm 2.2$ & $2.5 \pm 1.7$ & 0.053 \\
Basal FSH (IU/mL) & $6.3 \pm 3.2$ & $6.3 \pm 5.0$ & 0.959 \\
Total gonadotropin dose administered (IU) & $1869.9 \pm 481.4$ & $1809.4 \pm 372.2$ & 0.129 \\
Number of collected oocytes & $17.0 \pm 9.2$ & $20.0 \pm 11.5$ & 0.002 \\
Number of collected MII oocytes & $13.3 \pm 7.4$ & $15.2 \pm 8.5$ & 0.008 \\
Number of cryopreserved embryos & $8.8 \pm 4.5$ & $9.1 \pm 4.5$ & 0.381 \\
\hline
\end{tabular}

a Student's t test.

eDET, elective double-embryo transfer; eSET, elective single-embryo transfer; FSH, follicle-stimulating hormone; MII, metaphase II

Table II: Clinical outcomes of the study groups

\begin{tabular}{llll}
\hline & eDET & eSET & $\mathrm{p}^{\mathrm{a}}$ \\
\hline Number of transfers & 291 & 209 & \\
Implantation rate (\%) & $29.7 \%$ & $44.4 \%$ & $<0.001$ \\
Miscarriage rate (\%) & $19.4 \%$ & $21.7 \%$ & 0.669 \\
Ongoing pregnancy rate (\%) & $37.8 \%$ & $34.8 \%$ & 0.497 \\
Multiple pregnancy rate (\%) & $26.9 \%$ & $1.9 \%$ & $<0.001$ \\
\hline
\end{tabular}

${ }^{a}$ Continuity correction chi-square test.

eDET, elective double-embryo transfer; eSET, elective single-embryo transfer

Table III: Multiple linear regression model for evaluating the association of transferring two embryos in two sequential transfers (eSET + SET), compared with double-embryo transfer, with the chance of implantation (with adjustment for confounders)

\begin{tabular}{lllll}
\hline & Coefficient (B) & Standard error & $\mathrm{t}$ & $\mathrm{p}$ \\
\hline Constant & 0.515 & 0.169 & 3.056 & 0.002 \\
eSET + SET protocol & 0.142 & 0.036 & 3.942 & $<0.001$ \\
Women's age (years) & -0.008 & 0.004 & -1.885 & 0.060 \\
Number of cryopreserved embryos & 0.008 & 0.004 & 2.009 & 0.045 \\
\hline
\end{tabular}

eSET, elective single-embryo transfer; SET, single-embryo transfer 


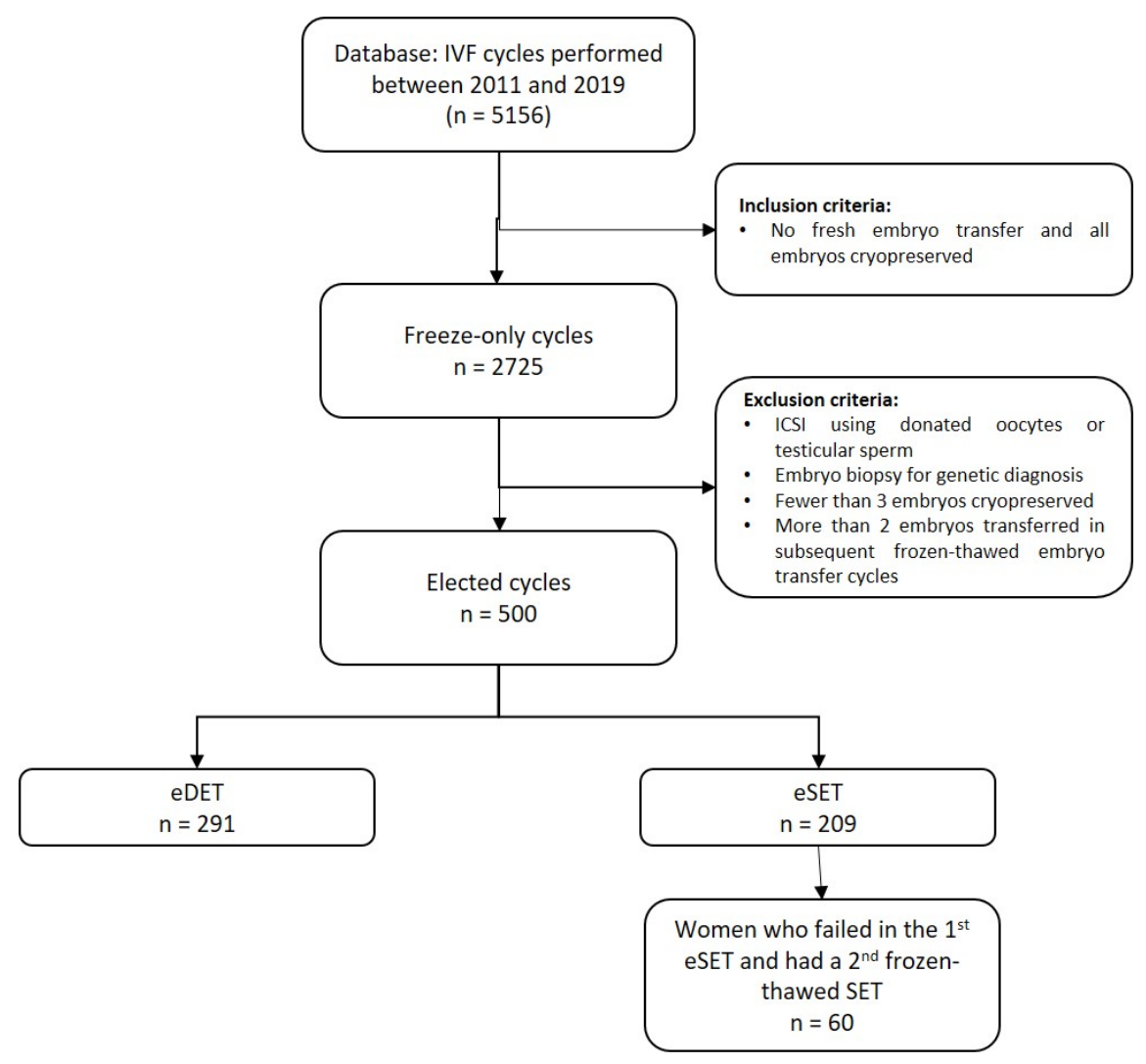

Estimated cumulative ongoing PR: 52\%

[ongoing PR for the $1^{\text {st }}$ SET + ongoing PR for the $2^{\text {nd }}$ SET * (1 - ongoing PR for the $1^{\text {tit }}$ SET)]
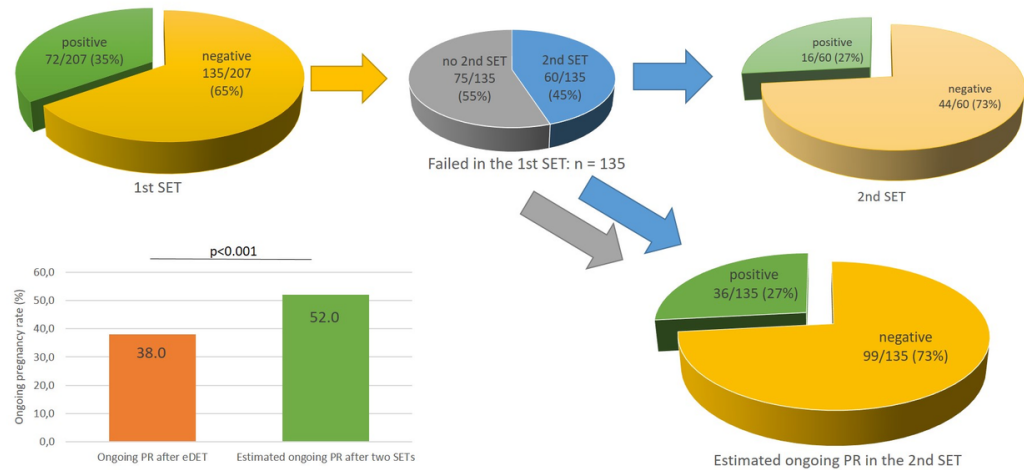